\title{
TIME-SCALE SEPARATION AND STATE AGGREGATION IN SINGULARLY PERTURBED SWITCHING DIFFUSIONS*
}

\author{
G. Yin and M. Kniazeva \\ Department of Mathematics \\ Wayne State University \\ Detroit, MI 48202 \\ gyin, maria@math.wayne.edu
}

\begin{abstract}
This work presents asymptotic results for singularly perturbed switching diffusions consisting of diffusion components and a pure jump component. The states of the pure jump component are divisible into a number of groups having recurrent states. An aggregated process is obtained by collecting all the states in each recurrent group as one state. We show the aggregated process converges weakly to a switching diffusion with generator being an average with respect to the quasi-stationary distribution of the jump process.
\end{abstract}

\section{INTRODUCTION}

Recent applications in manufacturing and queueing networks have posed many challenging problems involving singularly perturbed Markovian systems (see $[1,14,16,15,17,21,22]$ and the references therein). For example, in manufacturing systems, one often models the machine capacity by a Markov chain with finite state space to describe the situation that the machines are subject to breakdowns and repairs. Meanwhile, the demand of the underlying product may be considered as a diffusion process to capture the uncertainty of the demand behavior. A direct treatment of large dimensional systems is frequently infeasible from a computational point of view. A viable alternative calls for time-scale separation modeling and aggregation approach so as to break the system into small pieces with manageable size, which naturally leads to the formulation of singularly perturbed system.

In [9], we studied properties of probability distribution of fast varying Markov chain by matched asymptotic expansion. Chains with fast and slow motions

\footnotetext{
*This research was supported in part by the National Science Foundation under grant DMS9529738 , and in part by Wayne State University.
}

The original version of this chapter was revised: The copyright line was incorrect. This has been corrected. The Erratum to this chapter is available at DOI: 10.1007/978-0-387-35359-3_40 
were consider in [10]; further results were contained in [21, 23, 24] among others. We carried out further investigation to include singularly perturbed diffusion processes and singularly perturbed switching diffusions $[6,7,8,19,20]$. This paper continues our effort by treating singularly perturbed switching-diffusion processes with the jump process decomposed into several groups with fast and slow motions. The rest of the paper is arranged as follows. Section 2 gives the formulation. Section 3 presents a number of asymptotic results. Section 4 concludes the paper with brief remarks.

\section{FORMULATION}

Suppose $T>0$. We work with finite horizon $[0, T]$. Consider a nonstationary Markov process $Y^{\varepsilon}(t)=\left(X(t), \gamma^{\varepsilon}(t)\right)$, where $X(t)$ is a 1-dimensional diffusion process and $\gamma^{\varepsilon}(t)$ is a pure jump process.

The state space of the process $Y^{\varepsilon}(\cdot)$ is $\mathcal{X}=[0,1] \times \mathcal{M}=[0,1] \times\{1, \ldots, m\}$ (i.e., we consider the case of periodic diffusions with state space $[0,1]$ and the jump process has state space $\mathcal{M}$ ). If $\gamma^{\varepsilon}(t)=i$, the evolution of $X(t)$ is represented by the differential operator $\mathcal{D}_{i}$ with $\mathcal{D}_{i} \tilde{f}=(1 / 2) a_{i}(x, t)\left(\partial^{2} / \partial x^{2}\right) \tilde{f}+$ $b_{i}(x, t)(\partial / \partial x) \tilde{f}$ for appropriate smooth functions $\tilde{f}(\cdot), a_{i}(\cdot)$ and $b_{i}(\cdot)$. With $X(t)=x$ fixed, the pure jump component $\gamma^{\varepsilon}(t)$ satisfies $P\left(\gamma^{\varepsilon}(t+\delta)=j \mid \gamma^{\varepsilon}(t)=\right.$ $i, X(t)=x)=q_{i j}^{\varepsilon}(x, t) \delta+o(\delta)$, where $o(\delta) \rightarrow 0$ as $\delta \rightarrow 0$, for each $i, j \in$ $\mathcal{M}, q_{i j}^{\varepsilon}(x, t) \geq 0$ when $j \neq i$, and $q_{i i}^{\varepsilon}(x, t)=-\sum_{j \neq i} q_{i j}^{\varepsilon}(x, t)$. Assume that all coefficients $a_{i}(\cdot), b_{i}(\cdot)$, and $q_{i j}^{\varepsilon}(\cdot)$ are sufficiently smooth functions of $(x, t)$. In view of [5], for $i=1, \ldots, m$, and smooth real-valued function $f(\cdot, \cdot, i)$, the generator $L^{\varepsilon}$ of this switching-diffusion process takes the form

$$
\begin{aligned}
L^{\varepsilon} f(x, t, i)=\frac{\partial f(x, t, i)}{\partial t} & +b_{i}(x, t) \frac{\partial f(x, t, i)}{\partial x}+\frac{1}{2} a_{i}(x, t) \frac{\partial^{2} f(x, t, i)}{\partial x^{2}} \\
& +\sum_{j=1}^{m} q_{i j}^{\varepsilon}(x, t) f(x, t, j) .
\end{aligned}
$$

The probability density of the process, $p^{\varepsilon}(x, t)=\left(p_{1}^{\varepsilon}(x, t), \ldots, p_{m}^{\varepsilon}(x, t)\right)$ is the solution of the forward equation

$$
\frac{\partial p_{i}^{\varepsilon}}{\partial t}=\mathcal{D}_{i}^{*} p_{i}^{\varepsilon}+\sum_{j=1}^{m} p_{j}^{\varepsilon} q_{i j}^{\varepsilon}, p_{i}^{\varepsilon}(x, 0)=g_{i}(x), \quad i=1, \ldots, m,
$$

where $\mathcal{D}_{i}^{*}$ is the adjoint of $\mathcal{D}_{i}, \mathcal{D}_{i}^{*} \cdot=\mathcal{D}_{i}^{*}(x, t) \cdot=(1 / 2)\left(\partial^{2} / \partial x^{2}\right)\left(a_{i}(x, t) \cdot\right)-$ $(\partial / \partial x)\left(b_{i}(x, t) \cdot\right)$, and $g(x)=\left(g_{1}(x), \ldots, g_{m}(x)\right)$ is the initial distribution for $Y^{\varepsilon}(t)$.

Assume that $Q^{\varepsilon}(x, t)=\left(q_{i j}^{\varepsilon}(x, t)\right)$ has the form

$$
Q^{\varepsilon}(x, t)=\frac{1}{\varepsilon} \widetilde{Q}(x, t)+\widehat{Q}(x, t), \text { where } \widetilde{Q}(x, t)=\operatorname{diag}\left(\widetilde{Q}^{1}(x, t), \ldots, \widetilde{Q}^{l}(x, t)\right)
$$


such that for each $t \in[0, T]$, and each $k=1, \ldots, l, \widetilde{Q}^{k}(x, t)$ is a generator with dimension $m_{k} \times m_{k}$, The state space $\mathcal{M}$ of the jump process admits a decomposition

$$
\begin{aligned}
\mathcal{M} & =\mathcal{M}_{1} \cup \mathcal{M}_{2} \cup \ldots \cup \mathcal{M}_{l} \\
& =\left\{\varpi_{11}, \ldots, \varpi_{1 m_{1}}\right\} \cup\left\{\varpi_{21}, \ldots, \varpi_{2 m_{2}}\right\} \cup\left\{\varpi_{l 1}, \ldots, \varpi_{l m_{l}}\right\} .
\end{aligned}
$$

The basic assumptions we are using are as follows.

(C1) For each fixed $x \in[0,1], \gamma^{\varepsilon}(\cdot)$ is a Markov chain with state space $\mathcal{M}$.

(C2) For each $i=1, \ldots, m$,

- $a_{i}(\cdot) \in C^{2,1}$, (i.e., the second partial derivatives of $a_{i}(\cdot)$ with respect to $x$ and the partial derivatives with respect to $t$ are continuous), $b_{i}(\cdot) \in C^{1,1}$;

- $(\partial / \partial t) a_{i}(x, \cdot)$ and $(\partial / \partial t) b_{i}(x, \cdot)$ are Lipschitz on $[0, T]$;

$-a_{i}(x, t)>0$. for all $(x, t) \in[0,1] \times[0, T]$.

(C3) The function $\widetilde{Q}(\cdot, \cdot) \in C^{1,1}$. For each $x \in[0,1],(\partial / \partial t) \widetilde{Q}(x, \cdot)$ and $\widehat{Q}(x, \cdot)$ are Lipschitz continuous on $[0, T]$.

(C4) For each $x \in[0,1]$, each $t \in[0, T]$, and each $i=1, \ldots, l, \widetilde{Q}^{i}(x, t)$ is weakly irreducible, i.e.,

$$
\nu^{i}(x, t) \widetilde{Q}^{i}(x, t)=0, \nu^{i}(x, t) \mathbb{1}_{m_{i}}=\sum_{j=1}^{m_{i}} \nu_{j}^{i}(x, t)=1
$$

has a unique solution that is nonnegative (i.e., $\nu_{j}^{i}(x, t) \geq 0$ for each $j=$ $\left.1, \ldots, m_{i}\right)$ and is termed quasi-stationary distribution. In the above $\mathbb{1}_{m_{i}}$ is an $m_{i}$-dimensional column vector that has all components being equal to 1 .

For basic properties of Markov chains, see [2] via a piecewise-deterministic approach. In what follows, we obtain certain weak convergence results for the model posed. For discussion of weak convergence, see [4, 11]. Related studies about stochastic differential systems are in [18].

\section{ASYMPTOTIC PROPERTIES}

First, we obtain a result on the asymptotic expansion of the system of interest. The rest of the section mainly concerns about occupation measures. We derive results on a fixed- $x$ process. The idea behind is that when $x$ is fixed, the corresponding process is a Markov chain. Define $\widetilde{\mathbb{1}}=\operatorname{diag}\left(\mathbb{1}_{m_{1}}, \ldots, \mathbb{1}_{m_{l}}\right)$, and

$$
\bar{Q}(x, t)=\operatorname{diag}\left(\nu^{1}(t), \ldots, \nu^{l}(t)\right) \hat{Q}(x, t) \tilde{\mathbb{1}} .
$$




\subsection{Asymptotic Expansion}

The following lemma can be obtained by using the techniques of [20] (Note: $\widetilde{Q}(x, t) \widetilde{\mathbb{1}}=0)$. We omit the proof here.

Lemma 3.1. Assume (C1)-(C4). For $k=1, \ldots, l$, denote by $\nu^{k}(x, t)$ the quasi-stationary distribution of $\widetilde{Q}^{k}(t)$. Then the following assertions hold:

(i) For some $\kappa_{0}>0$,

$$
p^{\varepsilon}(x, t)=v(x, t) \operatorname{diag}\left(\nu^{1}(x, t), \ldots, \nu^{l}(x, t)\right)+O\left(\varepsilon+\exp \left(-\kappa_{0} t / \varepsilon\right)\right),
$$

where $v(x, t)=\left(v_{1}(x, t), \ldots, v_{l}(x, t)\right)$ satisfies

$$
\begin{aligned}
& \frac{\partial v(x, t)}{\partial t}=\overline{\mathcal{D}}^{*} v(x, t)+v(x, t) \bar{Q}(x, t) \\
& v(x, 0)=\left(g^{1}(x) \mathbb{1}_{m_{1}}, \ldots, g^{l}(x) \mathbb{1}_{m_{l}}\right)
\end{aligned}
$$

where $\overline{\mathcal{D}}^{*}$ is the "average" of the forward operators w.r.t. the quasi-stationary distribution (see $[19,20])$ with $\overline{\mathcal{D}}^{*} v(x, t)=\left(\overline{\mathcal{D}}_{1}^{*} v_{1}(x, t), \ldots, \overline{\mathcal{D}}_{l}^{*} v_{l}(x, t)\right)$ and $g^{i}(x) \in \mathbb{R}^{1 \times m_{i}}$ denotes the ith partitioned vector of $g(x)$.

(ii) For fixed $x \in[0,1]$, denote the transition probability by $P^{\varepsilon}\left(x, t, t_{0}\right)$. Then for some $\kappa_{0}>0, P^{\varepsilon}\left(x, t, t_{0}\right)=\bar{P}\left(x, t, t_{0}\right)+O\left(\varepsilon+\exp \left(-\kappa_{0}\left(t-t_{0}\right) / \varepsilon\right)\right)$, where (using the notation of partitioned matrices)

$$
\bar{P}\left(x, t, t_{0}\right)=\widetilde{\mathbb{1}} V\left(x, t, t_{0}\right) \operatorname{diag}\left(\nu^{1}(x, t), \ldots, \nu^{l}(x, t)\right),
$$

and $V\left(x, t, t_{0}\right)=\left(v_{i j}\left(x, t, t_{0}\right)\right) \in \mathbb{R}^{l \times l}$ satisfying the differential equation:

$$
\frac{\partial V\left(x, t, t_{0}\right)}{\partial t}=V\left(x, t, t_{0}\right) \bar{Q}(x, t), V\left(x, t_{0}, t_{0}\right)=I
$$

where $\bar{Q}(x, t)$ is defined in (1.4).

\section{$3.2 \quad$ Fixed- $x$ Processes}

To proceed, define the "fixed- $x$ " processes $\tilde{Y}^{\varepsilon}(t)$ and $\bar{Y}_{i}^{\varepsilon}(t)$ as follows. Denote

$$
\tilde{Y}^{\varepsilon}(t)=\left.Y^{\varepsilon}(t)\right|_{X(t)=x}=\left(x, \gamma^{\varepsilon}(t)\right), \quad \text { and } \bar{Y}_{i}^{\varepsilon}(t)=\left.Y^{\varepsilon}(t)\right|_{X(t)=x, \gamma^{\varepsilon}(t) \in \mathcal{M}_{i}} .
$$

For fixed $x$, define $\bar{\gamma}^{\varepsilon}(t)=i$, if $\gamma^{\varepsilon}(t) \in \mathcal{M}_{i}$, for $i=1, \ldots, l$. Finally define the aggregated process $\bar{Y}^{\varepsilon}(t)$ by $\bar{Y}^{\varepsilon}(t)=\left(X(t), \bar{\gamma}^{\varepsilon}(t)\right)$. For any $t \in[0, T], x \in[0,1]$, $i=1, \ldots, l$ and $j=1, \ldots, m_{i}$, define

$$
\eta_{i j}^{\varepsilon}(x, t)=E\left(\int_{0}^{t}\left(I_{\left\{\tilde{Y}^{e}(s)=\left(x, \varpi_{i j}\right)\right\}}-\nu_{j}^{i}(x, s) I_{\left\{\bar{Y}_{i}^{\varepsilon}(s)\right\}}\right) d s\right)^{2} .
$$

Proposition 3.2. Suppose that (C1)-(C4) hold. Then for $i=1, \ldots, l, j=$ $1, \ldots, m_{i}, \sup _{x \in[0,1], 0 \leq t \leq T} \eta_{i j}^{\varepsilon}(x, t)=O(\varepsilon)$. 
Sketch of Proof. Recall that for $i=1, \ldots, l, \bar{\gamma}^{\varepsilon}(t)=i$ iff $\gamma^{\varepsilon}(t) \in \mathcal{M}_{i}$. Differentiating $\eta_{i j}^{\varepsilon}(\cdot)$ with respect to $t$ leads to

$$
\begin{aligned}
\frac{\partial \eta_{i j}^{\varepsilon}(x, t)}{\partial t}=2\left[\int_{0}^{t} P\left(\tilde{Y}^{\varepsilon}(t)=\left(x, \varpi_{i j}\right), \tilde{Y}^{\varepsilon}(s)=\left(x, \varpi_{i j}\right)\right) d s\right. \\
-\int_{0}^{t} \nu_{j}^{i}(x, t) P\left(\bar{Y}_{i}^{\varepsilon}(t), \tilde{Y}^{\varepsilon}(s)=\left(x, \varpi_{i j}\right)\right) d s \\
\quad-\int_{0}^{t} \nu_{j}^{i}(x, s) P\left(\tilde{Y}^{\varepsilon}(t)=\left(x, \varpi_{i j}\right), \bar{Y}_{i}^{\varepsilon}(s)\right) d s \\
\left.+\int_{0}^{t} \nu_{j}^{i}(x, s) \nu_{j}^{i}(x, t) P\left(\bar{Y}_{i}^{\varepsilon}(t), \bar{Y}_{i}^{\varepsilon}(s)\right) d s\right] .
\end{aligned}
$$

We can show that

$$
\begin{gathered}
\int_{0}^{t} P\left(\tilde{Y}^{\varepsilon}(t)=\left(x, \varpi_{i j}\right), \tilde{Y}^{\varepsilon}(s)=\left(x, \varpi_{i j}\right)\right) d s-\int_{0}^{t} \nu_{j}^{i}(x, t) P\left(\bar{Y}_{i}^{\varepsilon}(t), \tilde{Y}^{\varepsilon}(s)=\left(x, \varpi_{i j}\right)\right) d s \\
=\int_{0}^{t} O\left(\varepsilon+\exp \left(-\kappa_{0}(t-s) / \varepsilon\right)\right) d s=O(\varepsilon), \text { and } \\
-\int_{0}^{t} \nu_{j}^{i}(x, s) P\left(\tilde{Y}^{\varepsilon}(t)=\left(x, \varpi_{i j}\right), \bar{Y}_{i}^{\varepsilon}(s)\right) d s+\int_{0}^{t} \nu_{j}^{i}(x, s) \nu_{j}^{i}(x, t) P\left(\bar{Y}_{i}^{\varepsilon}(t), \bar{Y}_{i}^{\varepsilon}(s)\right) d s \\
=\int_{0}^{t} O\left(\varepsilon+\exp \left(-\kappa_{0}(t-s) / \varepsilon\right)\right) d s=O(\varepsilon) .
\end{gathered}
$$

Therefore,

$$
\frac{\partial \eta_{i j}^{\varepsilon}(x, t)}{\partial t}=2 \int_{0}^{t} O\left(\varepsilon+\exp \left(-\kappa_{0}(t-s) / \varepsilon\right)\right) d s=O(\varepsilon) .
$$

This implies that $\eta_{i j}^{\varepsilon}(x, t)=O(\varepsilon)$ and the bound is uniform in $x$ and $t$.

\subsection{Weak Convergence of $\bar{Y}^{\varepsilon}(\cdot)$}

We aim at deriving a weak convergence result for $\bar{Y}^{\varepsilon}(\cdot)$. First, we obtain its tightness.

Proposition 3.3. Assume (C1)-(C4). Then $\bar{Y}^{\varepsilon}(\cdot)$ is tight in $D^{2}[0, T]$.

Proof. We use the Kurtz' criteria. Denote by $\mathcal{F}_{t}^{\varepsilon}$ the $\sigma$-algebra generated by $\left\{\bar{Y}^{\varepsilon}(u), u \leq t\right\}$ and $E_{t}^{\varepsilon}$ the conditional expectation on $\mathcal{F}_{t}^{\varepsilon}$. Then

$$
\begin{aligned}
E_{t}^{\varepsilon}\left|\bar{Y}^{\varepsilon}(t+s)-\bar{Y}^{\varepsilon}(t)\right|^{2} \leq & K E_{t}^{\varepsilon}[X(t+s)-X(t)]^{2}+2 E_{t}^{\varepsilon}\left[\bar{\gamma}^{\varepsilon}(t+s)-\bar{\gamma}^{\varepsilon}(t)\right]^{2} \\
& \leq K \sum_{k=1}^{m} E_{t}^{\varepsilon} E\left([X(t+s)-X(t)]^{2} \mid \mathcal{F}_{t}^{\varepsilon}, \gamma^{\varepsilon}(t)=k\right) \\
& +2 E_{t}^{\varepsilon} \int_{0}^{1} E\left(\left[\bar{\gamma}^{\varepsilon}(t+s)-\bar{\gamma}^{\varepsilon}(t)\right]^{2} \mid \mathcal{F}_{t}^{\varepsilon}, X(t)=x\right) d x .
\end{aligned}
$$


Note that for each $k=1, \ldots, m$, when $\gamma^{\varepsilon}(t)=k$,

$$
X(t)=X(0)+\int_{0}^{t} b_{k}(X(u), u) d u+\int_{0}^{t} \sqrt{a_{k}(X(u), u)} d w
$$

where $w(\cdot)$ is a standard Brownian motion. By virtue of the boundedness of $a_{k}(\cdot)$ and in view of a well-known result in stochastic calculus (see [13, p. 125]), $E_{t}^{\varepsilon}\left(\int_{t}^{t+s} \sqrt{a_{k}(x, u)} d w\right)^{2} \leq K s$. Owing to the defining equation (1.11), and using the boundedness of $b_{k}(\cdot), E\left([X(t+s)-X(t)]^{2} \mid \mathcal{F}_{t}^{\varepsilon}, \gamma^{\varepsilon}(t)=k\right) \leq$ $K\left(s^{2}+s\right)$.

As for the last term in (1.10),

$$
\begin{aligned}
& \int_{0}^{1} E\left(\left[\bar{\gamma}^{\varepsilon}(t+s)-\bar{\gamma}^{\varepsilon}(t)\right]^{2} \mid \mathcal{F}_{t}^{\varepsilon}, X(t)=x\right) d x \\
& \quad \leq K \sum_{i=1}^{l+1} \sum_{j=1}^{m_{i}} \int_{0}^{1} E\left(\left[\bar{\gamma}^{\varepsilon}(t+s)-\bar{\gamma}^{\varepsilon}(t)\right]^{2} \mid \gamma^{\varepsilon}(t)=\varpi_{i j}, X(t)=x\right) d x
\end{aligned}
$$

Note that

$$
\begin{aligned}
& E\left(\left[\bar{\gamma}^{\varepsilon}(t+s)-\bar{\gamma}^{\varepsilon}(t)\right]^{2} \mid \gamma^{\varepsilon}(t)=\varpi_{i j}, X(t)=x\right) \\
& \quad=\sum_{k=1}^{l}(k-i)^{2} P\left(\bar{\gamma}^{\varepsilon}(t+s)=k \mid \gamma^{\varepsilon}(t)=\varpi_{i j}, X(t)=x\right) \\
& \quad \leq l^{2} \sum_{k \neq i} v_{i k}(t+s, t, x)+O\left(\varepsilon+\exp \left(-\kappa_{0} s / \varepsilon\right)\right) .
\end{aligned}
$$

The definition of $V(t, s, x)$ then infers that for each $x \in[0,1], \lim _{s \rightarrow 0} v_{i k}(t+$ $s, t, x)=0$ for $i \neq k$. Putting the above arguments together, what we have shown is: $\lim _{s \rightarrow 0} \lim _{\varepsilon \rightarrow 0} E E_{t}^{\varepsilon}\left|\bar{Y}^{\varepsilon}(t+s)-\bar{Y}^{\varepsilon}(t)\right|^{2}=0$. Then the tightness criterion (see $\left[11\right.$, Theorem 3, p. 47]) infers that the process $\left\{\bar{Y}^{\varepsilon}(\cdot)\right\}$ is tight in $D^{2}[0, T]$.

Define an "averaged" generator by

$$
\bar{L} \tilde{g}(x, t, i)=\frac{\partial \tilde{g}(x, t, i)}{\partial t}+\overline{\mathcal{D}}_{i} \tilde{g}(x, t, i)+\sum_{j=1}^{l} \bar{q}_{i j}(x, t) \tilde{g}(x, t, j)
$$

for any bounded and measurable function $\tilde{g}(\cdot)$ satisfying $\tilde{g}(\cdot, \cdot, i) \in C^{2,1}$ and for $i=1, \ldots, l$, where $\overline{\mathcal{D}}_{i}$ denotes the adjoint of $\overline{\mathcal{D}}_{i}^{*}$. By using the characteristic function $E \exp \left(\iota\left(\tau_{1} X(t)+\tau_{2} \bar{\gamma}(t)\right)\right.$, where $\iota$ is the pure imaginary number with $\iota^{2}=-1$, we can show that the martingale problem with operator $\bar{L}$ has a unique solution for each initial condition.

Proposition 3.4. Under the $(\mathrm{C} 1)-(\mathrm{C} 4), \bar{Y}^{\varepsilon}(\cdot)$ converges weakly to $\bar{Y}(\cdot)$, a Markov process with $\bar{L}$ given by (1.14). 
The main idea of the proof involves the use of martingale problem formulation (see [11]), i.e., we show that $\bar{Y}(t)=(X(t), \bar{\gamma}(t))$ is a solution of the martingale problem with operator $\bar{L}$. To establish the desired result, we show that for any bounded and measurable function $f(\cdot)$ with $f(\cdot, \cdot, \gamma) \in C^{2,1}$ for each $\gamma \in \mathcal{M}$, $f(X(t), t, \bar{\gamma}(t))-\int_{0}^{t} \bar{L} f(X(s), s, \bar{\gamma}(s)) d s$ is a martingale. To do so, define $\bar{f}(x, t, \gamma)=\sum_{k=1}^{l} f(x, t, k) I_{\left\{\gamma \in \mathcal{M}_{k}\right\}}$, work with the function $\bar{f}(\cdot)$ instead, and show that $\bar{f}(X(t), t, \gamma(t))-\int_{0}^{t} \bar{L} \bar{f}(X(s), s, \gamma(s)) d s$ is a martingale. To accomplish this, we work with the pre-limit problem, and define a piecewise constant process $\widetilde{X}^{\varepsilon}(\cdot)$ by $\widetilde{X}^{\varepsilon}(t)=X\left(k \delta_{\varepsilon}\right)$ for $t \in\left[k \delta_{\varepsilon},(k+1) \delta_{\varepsilon}\right)$. We then show that the limit of $\bar{f}\left(X(t+s), t+s, \gamma^{\varepsilon}(t+s)\right)-\bar{f}\left(X(t), t, \gamma^{\varepsilon}(t)\right)-\int_{t}^{t+s} \bar{L} \bar{f}\left(X(u), u, \gamma^{\varepsilon}(u)\right) d u$ is the same as that of the process with $X(t)$ replaced by $\tilde{X}(t)$. This enables us to characterize the limit. More details can be found in [19].

\section{References}

[1] V. V. Anisimov, Switching processes: Averaging principle, diffusion approximation and applications, Acta Appl. Math. 40 (1995), 95-141.

[2] M. H. A. Davis, Markov Models and Optimization, Chapman \& Hall, London, 1993.

[3] S. D. Eidelman, Parabolic Systems, North-Holland, New York, 1969.

[4] S. N. Ethier and T. G. Kurtz, Markov Processes: Characterization and Convergence, J. Wiley, New York, 1986.

[5] I. I. Gihman and A. V. Skorohod, Theory of Stochastic Processes, III, Springer-Verlag, Berlin, 1979.

[6] A. M. Il'in, R. Z. Khasminskii and G. Yin, Singularly perturbed switching diffusions: rapid switchings and fast diffusions, preprint, 1997.

[7] R. Z. Khasminskii and G. Yin, Asymptotic series for singularly perturbed Kolmogorov-Fokker-Planck equations, SIAM J. Appl. Math. 56 (1996), 1766-1793.

[8] R. Z. Khasminskii and G. Yin, On transition densities of singularly perturbed diffusions with fast and slow components, SIAM J. Appl. Math. 56 (1996), 1794-1819.

[9] R. Z. Khasminskii, G. Yin and Q. Zhang, Asymptotic expansions of singularly perturbed systems involving rapidly fluctuating Markov chains, SIAM J. Appl. Math. 56 (1996), 277-293.

[10] R. Z. Khasminskii, G. Yin and Q. Zhang, Constructing asymptotic series for probability distribution of Markov chains with weak and strong interactions, Quart. Appl. Math. LV (1997), 177-200.

[11] H. J. Kushner, Approximation and Weak Convergence Methods for Random Processes, with Applications to Stochastic Systems Theory, MIT Press, Cambridge, MA, 1984. 
[12] O. A. Ladyzhenskaia, V. A. Solonnikov and N. N. Ural'tseva, Linear and quasi-linear equations of parabolic type, Trans. Math. Monographs, V. 23, Amer. Math. Soc., Providence, RI, 1968.

[13] R. S. Liptser and A. N. Shiryayev, Statistics of Random Processes I, Springer-Verlag, New York, 1997.

[14] Z. G. Pan and T. Başar, $H^{\infty}$-control of Markovian jump linear systems and solutions to associated piecewise-deterministic differential games, in New Trends in Dynamic Games and Applications, G. J. Olsder (Ed.), 6194, Birkhäuser, Boston, 1995.

[15] R. G. Phillips and P. V. Kokotovic, A singular perturbation approach to modelling and control of Markov chains, IEEE Trans. Automat. Control 26 (1981), 1087-1094.

[16] J. R. Rohlicek and A. S. Willsky, The reduction of perturbed Markov generators: An algorithm exposing the role of transient states, J. ACM 35 (1988), 675-696.

[17] S. P. Sethi and Q. Zhang, Hierarchical Decision Making in Stochastic Manufacturing Systems, Birkhäuser, Boston, 1994.

[18] A. V. Skorohod, Asymptotic Methods of the Theory of Stochastic Differential Equations, Trans. Math. Monographs, V. 78, Amer. Math. Soc., Providence, 1989.

[19] G. Yin, Weak convergence of an aggregated process arising from singularly perturbed switching diffusions, preprint, 1998.

[20] G. Yin and M. Kniazeva, Singularly perturbed multidimensional switching diffusions with fast and slow switchings, preprint, 1997.

[21] G. Yin and Q. Zhang, Continuous-time Markov Chains and Applications: A Singular Perturbations Approach, Springer-Verlag, New York, 1998.

[22] G. Yin and Q. Zhang (Eds.), Mathematics of Stochastic Manufacturing Systems, Proc. 1996 AMS-SIAM Summer Seminar in Applied Mathematics, Lectures in Applied Mathematics, Amer. Math. Soc., Providence, RI, 1997.

[23] Q. Zhang and G. Yin, A central limit theorem for singularly perturbed nonstationary finite state Markov chains, Ann. Appl. Probab. 6 (1996), 650-670.

[24] Q. Zhang and G. Yin, Structural properties of Markov chains with weak and strong interactions, Stochastic Process Appl. 70 (1997), 181-197. 